\title{
Sensor stability assurance problems and their relationship with the overall problems of providing system performance quality
}

\author{
Petr Mikhailov ${ }^{1, *}$, Zhomart Ualiyev $^{2}$ \\ ${ }^{1}$ Penza state technological University Penza, Russia \\ ${ }^{2}$ Satbayev University Almaty, Kazakhstan
}

\begin{abstract}
Research has been carried out in the field of ensuring the stability of transmitters, which are designed to work in extreme conditions. The complexity of the solved problems and the need for a systemic approach, including structural, material science, are noted. Approaches of leading foreign and domestic companies - manufacturers of sensors and measuring systems to issues of metrological reliability of measuring transducers have been analyzed. Ambiguity of interpretation of the term "stability" by various developers and manufacturers of sensors is shown. Most often, the accompanying documentation for sensors, as well as prospectuses and catalogs indicate a qualitative characteristic, and very rarely quantitative parameters. At the same time, international quality standards indicate the need to disclose qualitative indicators and translate them into quantitative data. The concept of ensuring stability of sensors during their life cycle, based on international quality standards and new design and technological solutions, is proposed. On the example of the beam sensitive element of the metal film pressure sensor, a specific effective technique for stabilizing the electrophysical characteristics of the film heterostructure is shown.
\end{abstract}

\section{Introduction}

\begin{abstract}
About relevance, novelty and complexity of any problem in the majority it is possible to judge by two quite objective criteria of [1]:

- references in publications in various information sources;

- level of established terminology

According to the first criterion, it follows that the more often certain issues are mentioned in publications, the more important they are for society. In this regard, stability issues are, according to publications, global in nature, since they cover almost all aspects of human existence, from the state and behavior of ecosystems to the management of artificial objects created by man. In the sources studied, one can note the important point that as systems and societies develop and become more complex, the problem of ensuring their stable functioning becomes more and more urgent.
\end{abstract}

\footnotetext{
* Corresponding author: pit_mix@mail.ru
} 
A study of the terminological aspect of the novelty and complexity of the problem of stability suggests that terminology in this direction has not yet been fully established, discussions are taking place, both on the pages of specialized journals and at international and regional conferences and symposia. An indication that terminology on this issue is in its infancy is the different interpretations of the same terms in different fields.

So, a group of terms: "stability," "instability," "stable," "unstable," "stabilization", "stabilize" is used both in everyday life to characterize social and state relations, and in science and technology. In particular, quite often "stabilization" is mentioned in the description of various processes and systems. Here are some specific examples of the use of these terms.

In the public-state system: stability of society; stability of public life; policies aimed at stabilizing the ruble; unstable salary; unstable inter-State relations.

Natural phenomena and processes: stabilization of atmospheric processes; stable average monthly temperature; unstable atmospheric front.

In process and production systems: stabilizing annealing; stabilizing aging; stable and unstable technology; stable yield of suitable ones; stable size variation; unstable tolerance; stable (unstable) operation of the machine.

In science and technology: frequency stabilization; stable amplitude; stabilizer, etc.

The above examples show that both qualitative meaning is invested in the concept of "stability" (most often it refers to social societies) and quantitative (the latter aspect most characterizes technical systems). The relationship and interrelation of qualitative and quantitative aspects of stability can be established by qualimetric methods [1] with the involvement of a relatively new mathematical apparatus - graph theory [2] and fuzzy set theory [3].

In technology, the group of terms "stability" is widely used in the theory of autoregulation systems $[4,5]$. So, stabilization systems are called automatic control systems that maintain a constant (in particular, zero) value of the controlled value.

With regard to technological systems, the term "technological process stability" was introduced by directive documents (GOST 15895-77 and ST SEV 547-84) and characterize the property of the technological process (TP), which determines the constancy of the probability distributions of its parameters over a certain time interval without outside interference.

In the measuring technique, the stability of the measuring means (MM) is interpreted as the quality of the MM, reflecting the invariability in time of its metrological properties.

So, in the recommendations for interstate standardization of RMG 29-99, which were adopted instead of the morally and conceptually outdated GOST 16263-70, two interpretations of the term "stability" as a qualitative characteristic of MM are given, and MM instability is adopted as a quantitative characteristic. It is noted that instability is determined based on long-term MM studies.

In the quality management systems of products that were developed and introduced in the USSR in the $80 \mathrm{~s}$, "stability" was associated with quality indicators, most often technical and economic.

A turning point that highlighted the importance of ensuring and maintaining the stability of processes, regardless of their internal content and specific focus, was the development and implementation of a group of standards on quality of ISO-9000 [6, 7]. They note that the most important aspect of quality is the quality due to product design and expressed by the design indicator, which affects the deliberate characteristics of the product within a given gradation, as well as the stability of the product in various production and use conditions. From this it follows that stability is an essential component of quality, and in it operational and production factors are closely interconnected. 
Quite often, the terminology of the "stability" group is used in the literature on microelectronics, devoted to issues of quality of microelectronic equipment, reliability and diagnostics of radioelectronic equipment. Thus, [8, 9] uses the wording: "...stability of the physical structure of large integrated circuit (LIC)," which, in the author's opinion, is determined by the physical and technological compatibility of the materials used and the reproducibility of TPs for the manufacture of large integrated circuits. Change of electrophysical characteristics (EPC) of electrical radio products as a result of external factors is defined as instability and degradation of electric parameters.

On the other hand, technological actions aimed at improving parameters of hybrid integrated circuit (HIC) elements are called stabilization and adjustment of parameters [10]. It should be noted that the technological aspect of the term "stabilization" is quite often mentioned in the special literature. In particular, in order to improve the quality of film resistors and control their properties (resistivity, temperature coefficient of resistance - TCR, level of internal voltages in films), stabilization of resistive film resistance is used, as a result of which high stability of film properties is achieved and reliability and stability of electrical characteristics of film resistors are improved.

During patent research, which is a mandatory stage in the development of new devices and technologies, it was revealed that from 10 to $20 \%$ (depending on the belonging to a class of inventions) of the declared goals and solved problems belong to the category "stability." This is particularly evident in international patent classification classes such as G01 and H01.

The stability of technical systems is included in their technical and physical characteristics along with such basic categories as weight, the number of degrees of freedom, etc., when classifying, creating databases and designing machines and mechanisms.

The problem of ensuring the stability of the products being developed, one way or another, is faced by all developers and manufacturers, but it is especially relevant in industries such as RCT, aviation, nuclear energy. This is primarily due to the need to assign a significant working resource, the transition to a new element base, increased levels of external influencing factors acting on devices, materials and components [11-13].

\section{Theoretical analysis}

In open directories for semiconductor devices, temporary drifts of parameters, with rare exceptions, are not indicated at all or are not always correctly indicated. For example, in [14], the formula for determining the instability of the output voltage is given

$$
K_{t}=\frac{\Delta \text { Uout }}{\text { Uout }} \cdot 100 \% \quad \mathrm{t}=0, \mathrm{t}=\mathrm{n}
$$

and specified as a percentage regardless of time.

An analysis of the catalogs of manufacturers of sensors and measuring systems showed that the vast majority of manufacturers do not indicate the values of the time stability of the parameters and only some (units), although they indicate, only qualitatively, and not quantitatively. In particular, in the catalogues of such well-known foreign firms as "Druck" (Great Britain), "Piezotronic" (USA), "SCHLUMBERGER" (France) and several others, neither qualitative nor quantitative characteristics of the stability of parameters are indicated at all. Firms such as Honeywell (USA) for a 136PC-156-220 type sensor normalizes stability for a year with a value of $\pm 0,15 \%$.

The French company "Elgas" in its catalogs defines stability in the form of \{"Longtermdrift" $<0.2 \%$ of the annualrange (temperature drift less than $0.2 \%$ per year)].

Endevko, which produces sensors for the aerospace industry, does not indicate a temporary drift in the characteristics of [15] in its prospectuses. 
In the catalogue of products of such a dominant organization in Russia for sensors and measuring systems as «NIIFI» (Penza) [16], stability is characterized only qualitatively, the terms are used: "long-term stability of metrological characteristics (MC)," "stability of characteristics in a wide temperature range," "stability of MC during life," "stability of parameters when exposed to vibrations, shocks, temperatures"

In avenues of sensors of "MIDA» (Ulyanovsk) it is written down ". in sensors of temperature the highly stable thermosensitive element which guarantees the minimum leaving of temperature throughout a long time (no more than $0,5^{\circ} \mathrm{C}$ in 8 years)" to [17] is used.

The Ulyanovsk Instrument Design Bureau in the information sheet on the PDI-1 pressure sensor gives stability for 1 year in the form of $2,7 \cdot 10^{-3} \mathrm{~kg} / \mathrm{cm}^{2}(0,2 \%)$.

Sensors with sensitive elements (SE) from silicon-on-sapphire (SOS) structures are widely used in Russia in the oil, gas, nuclear industry and energy. Such sensors are produced by enterprises of Chelyabinsk, Orel, Ulyanovsk under the brands «Metran», «MIDA», «Krios», «LHP», «STAK» [18]. There is no information about their time stability in the published materials for the indicated sensors, but fundamentally it cannot be high, since initially there are large mechanical stresses (MS) in the SE of "Silicon on sapphire". These stresses are caused by the significant difference in thermal expansion coefficients (TEC) between the silicon epitaxial film and the sapphire substrate. The high temperature of silicon film formation on sapphire causes the appearance after cooling of the structure of significant residual MS proportional to the difference in the thermal expansion coefficients of silicon and sapphire, as well as the temperature of film formation. As experimental studies have shown, these MS are quite significant, they change during the operation and storage of sensors. In addition, sensors with SE based on "Silicon on sapphire" are critical to the conditions of sensor attachment to the measurement object.

The most comprehensive reference book on sensors, released recently under the auspices of the Russian Space Agency, contains practically no information on their stability [19].

A rare exception was the information on the stability of resistors given in reference book [20], in which the stability characteristics are represented by two groups of parameters:

1. Minimum operating time in hours and resistance change of resistors during minimum operating time in $\%$.

2. Persistence period and resistance change of resistors to the end of persistence period in $\%$.

The lack and simply lack of information from manufacturers on the stability of the sensors they produce, in our opinion, is due to several main reasons:

- difficulty of correct confirmation of time stability of technical characteristics of sensors, which is associated with high time and economic costs;

- insufficient development of mathematical apparatus for creating long-term prognostic models.

The development of measurement means with guaranteed time stability involves the creation of mathematical and physical models capable of providing the developer with sufficient information on the state of the characteristics of the device either after a given time interval or in a specified time range. The difficulty of creating such models is that the number of parameters considered is too large, in addition, it is necessary to take into account all the operating modes of the device at one point or another during the entire life of the sensors. Therefore, in practice, when developing, there is an overestimation of the perceived external influencing factors (EIF), such as increased or decreased temperature, thermal impacts, periodic increases and decreases in humidity, the influence of aggressive media, etc. In such cases, the sensor is designed according to critical EIF values, although in fact the operating conditions are only critical for a short time compared to the total operating resource. 
In terms of rationality, such critical models, while capable of facilitating the development and design costs of the MI, are not able to accurately predict the state of the system or sensor at any given point in time. If you accurately describe the operating conditions of the measuring instrument (MI), which, in general, is almost impossible due to the random nature of external influences, then the developed model will be unstable. Therefore, it is advisable to consider the hierarchical structure of the measuring system or sensor, dividing them into separate subsystems and elements (matching amplifier, bridge circuit, compensation circuit, elastic element, tensoresistor, metallization, etc.) and model building for each part, identify the most stable and unstable elements and structures.

Thus, the problem of ensuring the stability of technical systems is an extremely important and urgent task, including design, technological, materials science, environmental and other aspects.

As one of the possible solutions to the stability issue applicable to metal film sensors, the concept of their development process, presented in the form of a structural diagram, is proposed (Fig. 1) [21]. This solution uses the concept of "quality loop" adopted by international and Russian quality standards. To maintain a given level of stability during the entire service life, feedback and corresponding adjustment and proactive actions are implemented [22].

In Fig. 1 the following designations are adopted: PQS physical quantity sensor, 1 estimation of external instability impacts on sensitive unit and PQS reliability and stability; 2 - sensitive unit and PQS stabilization and regulation; 3 - sensitive unit and PQS specification flexibility; 4 - abstract model development; 5 - Sensitive unit measuring feature shift; 6 - stability indicator specification; 7 - Stabilizing feedback implementation.

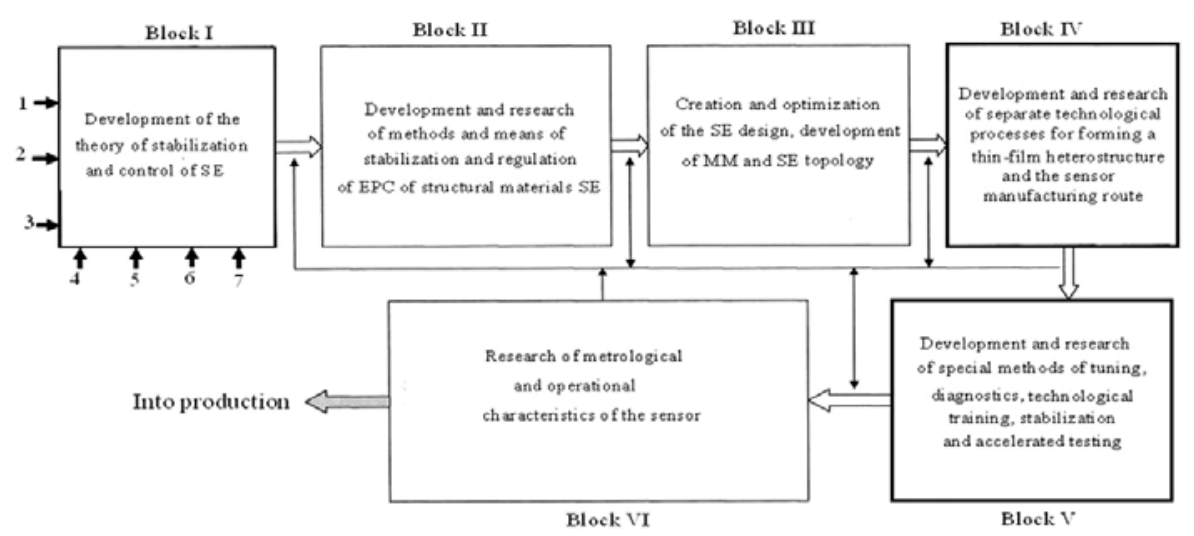

Fig. 1. Block diagram for development process of the highly stable physical quantity sensor (PQS) designed for extreme working conditions

An example of the practical implementation of the proposed concept is the beam SE of a metal-film pressure sensor made using planar-film technology - Figure 2. The SE is a complex structure in the form of a cross-shaped beam made as a whole with an annular support element. Tensostructures representing multilayer film compositions are formed on beams. They consist of insulating, adhesive, tensoresistive, contact and protective films 450$550 \mathrm{~nm}$ thick. Guaranteed stability of SE characteristics was achieved due to the selection of compatible structural and functional materials. In such materials, electrophysical characteristics remain stable over time and under the influence of external factors. When forming film structures, special stabilizing technological operations were used. These include thermal annealing in inert media, stabilization, training that helps to reduce internal stresses, streamline the structure and reduce the defects of films [23]. 


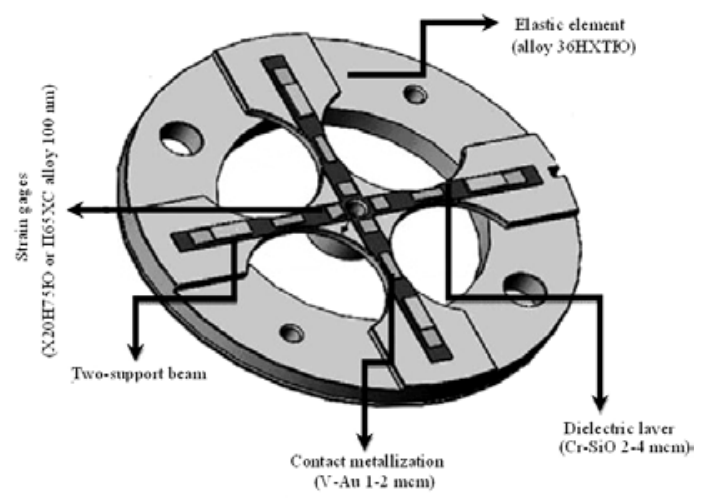

Fig. 2. Sensitive element of metal film pressure sensor based on cross beam

Among the developed structural and technological methods for ensuring guaranteed stability of metal film sensors, one can distinguish the main ones:

1. The use of heterogeneous film structures in which films have opposite TCR marks, with their total TCR close to zero in a certain temperature range (Fig. 3).

2. Development of controlled technologies for formation of metal-film heterogeneous structures in order to control their characteristics.

3. Application of special operations and regimes for stabilization and reduction of degradation processes.

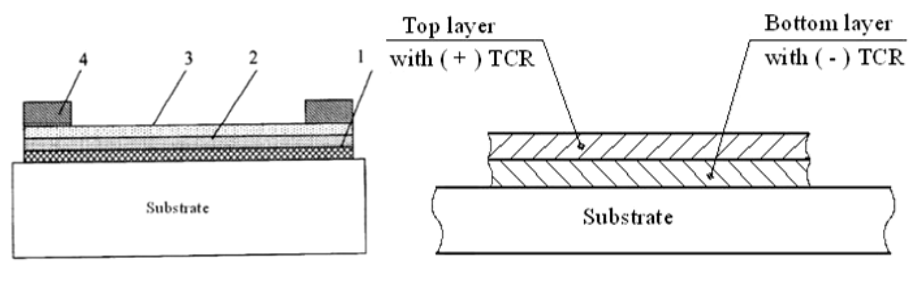

a

$\mathrm{b}$

Fig. 3. Heterogeneous structures of metal film sensor a - SE with layers: 1-dielectric, 2-resistive, 3protective, 4-contact; b-double-layer structure of tensoresistive film

Let's consider the choice of stable materials of the heterostructure of the metal film sensor.

\section{Selection of material and methods for producing thin film strain gauges}

The high reliability, time and temperature stability of the thin film transducers depend to a large extent on the properties of the strain gauges of the layers to be deposited.

Tensoresistors are subject to a number of specific requirements that distinguish them from resistors used in microelectronics. So, they should not change their characteristics when exposed to multiple and alternating mechanical loads in a given temperature range; they must have high temperature stability, and their temperature coefficient of resistance should not exceed $\pm 5 \cdot 10^{-5} 1 /{ }^{\circ} \mathrm{C}$; temporary stability of strain gauges should be at least $0.1 \%$ per 10 hours.

As tensoresistor materials from the tested group (X20H80, X20H75Ю, П65XC, К50C, tungsten-rhenium, molybdenum-rhenium), it is advisable to use alloys with a high recrystallization temperature (Table 1). The resistive layer is the most responsible, since it is the layer from which the measuring circuit and the resistor itself are formed. Very stringent requirements are placed on it: maximum coefficient of strain sensitivity; high mechanical 
characteristics, not lower than that of EE; high resistivity; high reproducibility of characteristics; very high temporary stability, which should not exceed $0.5 \%$ during continuous operation; have good adhesion with dielectric layer and material of contact groups; low value of temperature resistance coefficient; wide operating temperature range.

Very often, X20H80 sputtering is used for this layer, which, having high processability during the sputtering process. Has quite satisfactory performance characteristics: continuous operation time is more than 10 years, temporary instability is in the range of $2-2.5 \%$ per year for a separate strain gauge and up to $1-1.5 \%$ per year for strain gauges assembled in a bridge measuring circuit, operating temperature range from minus $80{ }^{\circ} \mathrm{C}$ to $100{ }^{\circ} \mathrm{C}$, in combination with $\mathrm{EE}$ of dispersion hardening alloys, the basic measurement error is within $0.5-1.0 \%$.

For tougher operating conditions, $\mathrm{X} 20 \mathrm{H} 75 \mathrm{H}$ alloy is used, which has higher performance characteristics: temporary instability is in the range of $1-1.5 \%$ per year for an individual strain gauge and $0.5-0.8 \%$ per year for strain gauges assembled in a bridge measuring chain, the temperature coefficient of resistance does not exceed $5 \cdot 10^{-4} 1 /{ }^{\circ} \mathrm{C}$.

One recent development of the strain-resistant alloy is the silicide-based alloy $\Pi 65 \mathrm{XC}$. Being the least technological of the listed materials, it has very high metrological characteristics: temporary instability does not exceed $0.1-0.2 \%$ per year, the TCR does not exceed $1 \cdot 10^{-4} 1 /{ }^{\circ} \mathrm{C}$, operating temperature range from cryogenic media to $300{ }^{\circ} \mathrm{C}$.

Alloy $\Pi 65 \mathrm{XC}$ is the most promising material for tensoresistors in the development of high-precision pressure sensors.

Table 1. Characteristics of tensoresistive materials

\begin{tabular}{|c|c|c|c|c|c|}
\hline \multirow{2}{*}{ Alloy grade } & \multirow{2}{*}{ Chemical composition $(\%)$} & \multicolumn{4}{|c|}{$\begin{array}{l}\text { Electrophysical characteristics of tensoresistive } \\
\text { alloys }\end{array}$} \\
\hline & & $\begin{array}{ll}\rho_{\mathrm{v}}, & (\mu \Omega \\
\mathrm{cm})\end{array}$ & S & $\begin{array}{l}\mathrm{TCR} \times 10^{-6}, \\
\left(1 /{ }^{\circ} \mathrm{C}\right)\end{array}$ & $\begin{array}{l}\text { TKLE } \times 10^{-5} \\
\left(1 /{ }^{\circ} \mathrm{C}\right)\end{array}$ \\
\hline Х20H75Ю & Cr 20, Ni 75, Al 5 & 14,0 & $1,8 \ldots 1,9$ & 50,0 & 1,5 \\
\hline П65ХС & $\begin{array}{l}\text { Cr 65, Si 31, Fe 1,5, Al 0,7, } \\
\text { La 0,3, Yt 0,3 }\end{array}$ & (powder) & $1,9 \ldots 2,2$ & 20,0 & - \\
\hline
\end{tabular}

The materials of electrical contacts of sensors are subject to a number of requirements, the main of which are good electrical and thermal conductivity, corrosion resistance, stability during long-term storage and operation. The latter factor is especially important for high temperature sensors, since temperature exposure can cause, firstly, a noticeable diffusion of atoms at the contact-resistor boundary, and secondly, lead to the formation of new phases in low-component materials; thirdly, lead to recrystallization of the material.

Only gold has such properties to the greatest extent. Despite the fact that the use of gold complicates the manufacturing process of sensors and increases their cost, there is no alternative to gold in operation in the range from cryogenic temperatures to $300{ }^{\circ} \mathrm{C}$.

\section{Conclusion}

Studies were carried out on the problem of ensuring the stability of the characteristics of metal film sensors, which showed that:

- when manufacturing sensors intended for operation in extreme operating conditions, it is necessary to introduce special technological operations to minimize drift of electrophysical characteristics due to external factors;

Such special operations include: thermal annealing of formed films, multiple thermal cycling, SE and sensors, accelerated tests;

The concept of ensuring the stability of sensors during their life cycle based on the "quality loop" is proposed. 
Design of SE beam designed for pressure sensors operating under extreme external factors is considered.

\section{References}

1. A. Subetto, Qualimetry: small encyclopedia, p. 244, (2015).

2. F. Harari, Graph Theory, p. 296 (2003)

3. L. Zade, The concept of a linguistic variable and its application to the adoption of approximate decisions, p. 165 (1976).

4. V. Doroshevich, Nano- and microsystem technology 9, pp. 21-23 (2008)

5. L. Koledov, Technology and designs of microcircuits, microprocessors and microassemblies, p. 394 (2008).

6. GOST 15467-79: Product quality management. Basic concepts. Terms and Definitions.

7. GOST R ISO 9000 - 2001 Quality management systems. Fundamentals and vocabulary.

8. A. Kovalenko, M. Petropavlovsky, Fundamentals of microelectronics: textbook. Manual, p. 240 (2008).

9. K. Ozhikenov, P. Mikhailov, A. Kabdoldina, Zh. Ualiyev, International Journal of Mechanical Engineering and Technology 9(10), pp. 1356-1367 (2018).

10. L. Gursky, V. Zelenin, A. Zhebin et al., Structure, topology and properties of film resistors (1988).

11. P. Mikhaylov, A. Kassimov, M. Khizirova, IJBR ISSN 0976-2612, v 8, pp. 152-157 (2017).

12. P. Mikhailov, B. Chuvykin, A. Mikhailov, Scientific-Technical Conference on Actual Problems of Electronic Instrument Engineering (A PEIE) (2020)

13. L. Zhao, Y. Zhao, Journal of Physics: Conference Series, v 48. pp. 178-183 (2006).

14. K. Polyanin Integrated voltage stabilizers, p. 192 (1979).

15. Catalog of products of the company "Endevco" www.endevco.com.

16. Catalog "Sensors and converting equipment" RCA, NIIFI http://www.niifi.ru.

17. http://www.midaus.com/images/docs/katalog.pdf.

18. http://www.metran.ru/home/pr/pdf/1dd.pdf

19. E. Bagdatev, A. Gorish, Ya. Malkov, Sensors of thermophysical and mechanical parameters. v. 2 (1998).

20. Yu. Andreev, D. Ivanov et al Resistors: (reference book) p. 352 (1981).

21. P. Mikhailov, K. Ozhikenov, A. Kasimov, A. Analieva, Bulletin of the Southern Federal University. Technical science, 3 (164) pp. 184-193 (2015).

22. ISO 9004-1-94 Quality management and quality system elements. Part 1. Guidelines.

23. C. Hsieh, C. Hung and Y. Li, Modern Mechanical Engineering, v. 03, pp. 104-113 (2013) 